\title{
NÚMERO ÓTIMO DE CLASSIFICAÇÕES INDEPENDENTES COM ERRO NA AVALIAÇÃO DA CONFORMIDADE DE PRODUTOS
}

\author{
Roberto da Costa Quinino * \\ Departamento de Estatística - ICEX \\ Universidade Federal de Minas Gerais \\ Belo Horizonte - MG \\ E-mail: roberto@est.ufmg.br \\ Emílio Suyama \\ Departamento de Estatística - ICEX \\ Universidade Federal de Minas Gerais \\ Belo Horizonte - MG \\ E-mail: suyama@est.ufmg.br \\ * Corresponding author/autor para quem as correspondências devem ser encaminhadas \\ Recebido em 10/2000, aceito em 05/2002 após 2 revisões
}

\section{Resumo}

Neste trabalho estudamos o número de ótimo de classificações independentes por unidade produzida supondo que haja dois tipos de erro: o tipo I no qual classifica-se um produto como não-conforme quando ele na realidade não é e o tipo II no qual classifica-se um produto como conforme quando na realidade é não-conforme. Desenvolvemos um modelo econômico para minimizar o custo médio total em função dos erros de classificação e do custo de cada classificação.

Palavras-chave: controle de atributos; erros de classificação; custo médio total.

\begin{abstract}
In this paper we study the optimal number of independent classifications on each produced unit when there are two types of classification errors: the type I error where a product is classified as non-conform when actually it is conform, and the type II error where a product is classified as conform when actually it isn't. We develop an economic model to minimize the total expected cost as a function of the classification errors and the cost of each classification.
\end{abstract}

Keywords: control of attributes, misclassification, total expected cost. 


\section{Introdução}

Freqüentemente, as características de qualidade não podem ser representadas de maneira conveniente numa forma numérica. Em tais casos é razoável classificar cada produto (ou característica de cada produto) inspecionado como conforme ou não-conforme. Essa é a situação quando a qualidade em consideração é o alinhamento de um eixo, a verificação da soldagem das junções de um circuito integrado ou a verificação de unidades de discos rígidos. Tratam-se de situações comuns no controle estatístico da qualidade para atributos. $\mathrm{O}$ problema é que a inspeção é realizada supondo que o sistema de classificação é perfeito, apesar de nem sempre podermos considerar essa hipótese como verdadeira. Burke et al. (1995), Gramopadhye et al. (1996), Johnson et al. (1991) e Kemp \& Kemp (1988) mostram e exemplificam que os erros de classificação podem comprometer seriamente o processo de avaliação da qualidade de atributos.

Neste trabalho tratamos de testes cujas classificações podem apresentar erros. Estamos considerando que dois tipos de erros são possíveis: um é o erro de classificação tipo II no qual classifica-se um produto como conforme sendo na realidade não-conforme; outro é o erro de classificação tipo I no qual classifica-se um produto como não-conforme sendo na realidade conforme. A questão aqui será de como melhorar o desempenho dos testes de qualidade através da diminuição da influência dos erros de classificação.

Greenberg \& Stokes (1995) apresentam um modelo que pode ser adaptado para determinar o número ótimo de classificações repetidas considerando apenas a presença do erro tipo I. Quinino et al. (1999) sugerem realizar $m(m \geq 0$ e inteiro) classificações independentes por produto fabricado e considerar como classificação final do produto aquela que corresponder à maioria dos resultados obtidos. Elaboram uma função de custo e propõem um método de determinação do valor de $m$ que minimize o custo esperado. Neste trabalho modificamos o critério de decisão de Quinino et al. (1999) de tal forma a diminuir o custo médio esperado. Além disso, propomos um critério de busca exaustiva com um limitante superior para determinação dos parâmetros ótimos, ideais no sentido econômico, menor do que aqueles discutidos por Quinino et al. (1999).

A estratégia que propomos para resolver o problema apresentado é realizar $m$ ( $m \geq 0$ e inteiro) classificações independentes por produto fabricado e considerar o produto conforme se o número de classificações independentes conformes for superior ao valor $a=\lfloor b m\rfloor$, onde $b$ é uma variável auxiliar com $0 \leq b<1$ e $\lfloor b m\rfloor$ indica o maior inteiro inferior ou igual a $b m$. Tal procedimento pode minimizar as classificações incorretas, mas também pode tornar o processo economicamente inviável. Sendo assim, há necessidade de um modelo que indique qual o número ótimo de classificações independentes repetidas e o respectivo critério de decisão.

O restante do trabalho é dividido em três seções. A seção 2 descreve o modelo probabilístico adequado. Na seção 3, discutimos o modelo de custo para determinação do número ótimo de classificações repetidas independentes. Derivamos um limitante superior para determinação do número ótimo de classificações repetidas. Uma aplicação numérica com implementação computacional do modelo proposto é apresentada na seção 4. Finalizamos o trabalho com algumas discussões que podem ser relevantes para novas aplicações e trabalhos futuros. 


\section{Modelo Probabilístico}

Suponha que $n$ produtos sejam classificados em conforme ou não-conforme. Seja $p$ a probabilidade de que um produto qualquer seja fabricado conforme, $e_{1}$ a probabilidade de que um produto conforme seja classificado como não-conforme em uma única classificação e $e_{2}$ a probabilidade de que um produto não-conforme seja classificado como conforme em uma única classificação.

Considere que cada um dos $n$ produtos seja classificado independentemente $m$ vezes. Considere $C_{i j}(i=1, \ldots, n ; j=1, \ldots, m)$ como uma variável $0-1$, correspondente ao $i j$-ésimo evento, isto é, a $j$-ésima classificação do $i$-ésimo produto; $C_{i}$ também uma variável $0-1$, correspondente à classificação final do $i$-ésimo produto após as $m$ classificações independentes.

Neste trabalho, estaremos considerando que o $i$-ésimo produto será julgado conforme, $C_{i}=1$, se e somente se $\sum_{j=1}^{m} C_{i j}>a, \mathrm{i}=1, \ldots, \mathrm{n}$. Quinino et al. (1999) consideram o produto conforme se $\sum_{j=1}^{m} C_{i j}>\lfloor 0,5 m\rfloor, \mathrm{i}=1, \ldots, \mathrm{n}$. Assim, trata-se de um caso particular do modelo que estamos aqui propondo $\operatorname{com} b=0,5$.

Os resultados tipo Bernoulli $C_{i}$ são independentes e identicamente distribuídos. Definindo $E_{i}$ $(i=1, \ldots, n)$ como uma variável $0-1$, correspondente ao estado real de fabricação do $i$-ésimo produto e utilizando o teorema da probabilidade total podemos expressar $\operatorname{Pr}\left[C_{i}=1\right]$ da seguinte forma:

$$
\begin{aligned}
\operatorname{Pr}\left[C_{i}=1\right] & =\operatorname{Pr}\left[E_{i}=1\right] \operatorname{Pr}\left[C_{i}=1 / E_{i}=1\right]+\operatorname{Pr}\left[E_{i}=0\right] \operatorname{Pr}\left[C_{i}=1 / E_{i}=0\right] \\
& =p\left[\sum_{x=0}^{a}\left(\begin{array}{l}
m \\
x
\end{array}\right) e_{1}^{x}\left(1-e_{1}\right)^{m-x}\right]+(1-p)\left[1-\sum_{x=0}^{a}\left(\begin{array}{l}
m \\
x
\end{array}\right) e_{2}^{x}\left(1-e_{2}\right)^{m-x}\right]
\end{aligned}
$$

Utilizando a notação $B[d ; e ; f]$ para uma função distribuição Binomial, com parâmetros $d$ e $e$, calculada no ponto $f$, temos

$$
\operatorname{Pr}\left[C_{i}=1\right]=p B\left[m ; e_{1} ; a\right]+(1-p)\left(1-B\left[m ; e_{2} ; a\right]\right)
$$

\section{Modelo para Minimização de Custos}

Consideremos $c_{0}$ o custo de classificar um produto uma única vez, $c_{1}$ o custo de erroneamente julgar um produto conforme como não-conforme e $c_{2}$ o custo de julgar erroneamente um produto não-conforme como conforme. Assim, se não houver classificações $(m=0)$, o custo médio total $\left(E_{m, a}\right)$ é dado por

$$
E_{m, a}\left(p, e_{1}, e_{2}\right)=n(1-p) c_{2}, m=0 .
$$

Em síntese, estamos considerando que toda a produção é conforme e por conseqüência somente o erro tipo II pode estar presente. O número médio de produtos não-conformes no mercado será $n(1-p)$.

Já para $n$ produtos, cada um classificado $m>0$ vezes, podemos expressar o custo médio total $\left(E_{m, a}\right)$ como 


$$
\begin{aligned}
E_{m, a}\left(p, e_{1}, e_{2}\right) & =n m c_{0}+n \operatorname{Pr}\left[E_{i}=1\right] \operatorname{Pr}\left[C_{i}=0 / E_{i}=1\right] c_{1}+n \operatorname{Pr}\left[E_{i}=0\right] \operatorname{Pr}\left[C_{i}=1 / E_{i}=0\right] c_{2} \\
& =n m c_{0}+n p B\left[m ;\left(1-e_{1}\right) ; a\right] c_{1}+n(1-p)\left(1-B\left[m ; e_{2} ; a\right]\right) c_{2}, m \geq 1 \mathrm{e} 0 \leq \mathrm{a}<\mathrm{m} .
\end{aligned}
$$

onde $B\left[m ;\left(1-e_{1}\right) ; a\right]=F_{1}$ representa a probabilidade de considerar erroneamente um produto conforme como não-conforme e $1-B\left[m ; e_{2} ; a\right]=F_{2}$ representa a probabilidade de considerar um produto não-conforme como conforme.

O problema que estamos considerando se reduz a determinar os valores de $\left(m^{\circ} ; a^{\circ}\right)$ que satisfaçam a equação (2.5) ou semanticamente, os valores $\left(m^{\circ} ; a^{\circ}\right)$ cujo custo médio total $\left(E_{m, a}\right)$ seja mínimo, considerando todos os $m$ e $a$ válidos.

$$
\left(m^{\circ} ; a^{\circ}\right)=\arg \min _{\substack{m, a \in Z^{+} \\ a=\lfloor b m\rfloor}}\left\{E_{m, a}\left(p, e_{1}, e_{2}\right)\right\}
$$

A solução de (2.5) é bastante complicada uma vez que (2.4) é discreta e possui somatórios dependentes de $m$. Tudo isto nos levou a desenvolver um procedimento exaustivo. Por sua característica intrínseca, os procedimentos exaustivos devem trabalhar juntamente com limitantes, fazendo com que a busca se processe em um intervalo finito de pontos. Assim sendo, nas proposições que seguem, reduziremos o número de candidatos aptos para $m^{\circ}$ e conseqüentemente para $a^{\circ}$ uma vez que $a^{\circ}=b m^{\circ}$.

Para erros de inspeção muito pequenos, o valor de $m$ que minimiza (2.5) será $m=0$ ou $m=1$, conforme a proposição 1 .

Proposição 1. Se os erros de inspeção são muito pequenos, ou seja, $e_{1}, e_{2} \rightarrow 0$, então $m^{\circ}=0$ ou $m^{\circ}=1$.

Prova. Para $m=0, E_{0,0}(p, 0,0)=n(1-p) c_{2}$. Para $m \geq 1, E_{m, a}(p, 0,0)=n m c_{0}$ pois $F_{1}=0$ e $F_{2}=1$. Portanto, o menor valor de $E_{m, a}(p, 0,0), m \geq 1$, é $E_{1,0}(p, 0,0)=n c_{0}$. Logo, se $E_{0,0}(p, 0,0) \leq E_{1,0}(p, 0,0)$ então $m^{\circ}=0$; caso contrário, $m^{\circ}=1$.

Devemos observar que $E_{m, a}(p, 0,0)$ e $E_{m, a}(p, 1,1)$ indicam custos associados respectivamente a um sistema classificador sem erros e a um sistema classificador onde se erra sempre. Desta forma podemos interpretá-los respectivamente como limitantes inferior e superior para $E_{m, a}\left(p, e_{1}, e_{2}\right)$.

Outro ponto importante a observar, proposição 2, é que se o valor $m=0$ minimiza $E_{m, a}(p, 0,0)$, então minimiza também $E_{m, a}\left(p, e_{1}, e_{2}\right)$. Em conjunto com a proposição 1 significa que se $(1-p) c_{2}<c_{0}$ então $m^{\circ}=0$, quando não é viável, considerando o custo médio total, realizar qualquer plano de inspeção.

Proposição 2. Se o valor $m=0$ minimiza $E_{m, a}(p, 0,0)$, então minimiza também $E_{m, a}\left(p, e_{1}, e_{2}\right)$.

Prova. Para $m \geq 1, E_{m, a}\left(p, e_{1}, e_{2}\right)>E_{m, a}(p, 0,0)$, e de $E_{0,0}\left(p, e_{1}, e_{2}\right)=E_{0,0}(p, 0,0)$, resulta que $E_{m, a}\left(p, e_{1}, e_{2}\right)-E_{0,0}\left(p, e_{1}, e_{2}\right)>E_{m, a}(p, 0,0)-E_{0,0}(p, 0,0) ;$ o lado direito é não negativo se o valor 0 minimiza $E_{m, a}(p, 0,0)$. 
Diversos limitantes são possíveis de serem gerados para $\left(m^{\circ} ; a^{\circ}\right)$, dependendo das hipóteses assumidas com relação ao modelo e seus parâmetros. A proposição 3 abaixo fornece um limitante bastante aberto, no sentido de que nenhuma hipótese foi considerada na sua elaboração.

Proposição 3. Existe um limitante superior para $m^{\circ}$ dado por:

$$
m^{\circ} \leq \frac{(1-p) c_{2}}{c_{0}} .
$$

Prova. Queremos $m^{\circ}$ tal que $E_{m^{\circ}, a^{\circ}}\left(p, e_{1}, e_{2}\right) \leq E_{m, a}\left(p, e_{1}, e_{2}\right), \quad m \geq 0$. Mas $E_{m^{\circ}, a^{\circ}}(p, 0,0) \leq E_{m^{\circ}, a^{\circ}}\left(p, e_{1}, e_{2}\right)$. Assim, $\quad E_{m^{\circ}, a^{\circ}}(p, 0,0) \leq E_{0,0}\left(p, e_{1}, e_{2}\right)$, ou seja $n m^{\circ} c_{0} \leq n(1-p) c_{2} \Rightarrow m^{\circ} \leq(1-p) c_{2} / c_{0} \quad \square$

O limitante proposto por Quinino et al. (1999) foi $m^{\circ} \leq 1+\left[p c_{1}+(1-p) c_{2}\right] / c_{0}$. Assim, facilmente observamos que este é superior ao definido em (2.6). O problema consiste então em verificar qual $m$ e $a$, que satisfazem (2.5) e (2.6).

\section{Aplicação Numérica}

O exemplo descrito a seguir é uma adaptação de uma consultoria, realizada em 1997, para uma grande empresa multinacional do setor de eletrônicos, com filial no Brasil, que fabrica placas-mãe para computadores. O modelamento inicial foi baseado em Greenberg \& Stokes (1995) e discutido em Quinino et al. (1999). A empresa fabricava 1000 placas-mãe por dia. Segundo os Engenheiros da empresa, o sistema produtivo não era eficiente quando comparado ao padrão internacional. Apresentava um percentual de placas defeituosas de 5\% contra uma média internacional de $2 \%$.

Estes fatos geravam reclamações dos clientes e, conseqüente, reposição das peças defeituosas. Dados históricos mostravam que a perda de clientes era mínima e a demanda apresentava um aumento contínuo. Isto era decorrente do baixo preço adotado quando comparado ao dos concorrentes. O objetivo da empresa a curto prazo era minimizar os custos globais originados com a reposição das placas defeituosas. Neste sentido, a gestão estratégica da qualidade não era foco naquela ocasião. $\mathrm{O}$ custo médio com a reposição das placas defeituosas era de 6.000,00 dólares diários o que representava um custo de 120,00 dólares por placa reposta.

Para tentar minimizar os custos de reposição, uma empresa de consultoria foi contratada. Esta sugeriu que fossem realizados testes automáticos em todas as placas já produzidas. A estratégia foi aceita pela empresa que dispunha de tecnologia implantada capaz de realizar testes não totalmente eficientes. Estes apresentavam um grau de acerto de $90 \%$ tanto para placas defeituosas ou não. $\mathrm{O}$ custo de cada teste era de 1,00 dólar e foi estimado pela empresa que placas em bom estado julgadas defeituosas representavam um custo de 80,00 dólares por placa. Adicionalmente, os testes podiam ser realizados repetidas vezes para uma mesma placa.

No contexto deste trabalho podemos então considerar $p=0,95$ a probabilidade de que uma placa-mãe seja fabricada conforme, $e_{1}=0,10$ a probabilidade de que uma placa-mãe 
conforme seja classificada como não-conforme em uma única classificação e $e_{2}=0,10$ a probabilidade de que uma placa-mãe não-conforme seja classificada como conforme em uma única classificação.

Os responsáveis pelo setor de planejamento e produção concordaram que o problema se resumia em determinar o número ideal de classificações repetidas independentes $(m)$ para as placas-mãe bem como o valor que define o critério de decisão $(a)$. O objetivo era minimizar o custo médio total da produção diária. Para tanto, o departamento financeiro da empresa confirmou os custos $c_{0}=\$ 1,00, c_{1}=\$ 80,00$ e $c_{2}=\$ 120,00$.

Como $(1-p) c_{2}>c_{0}$ concluímos que o planejamento com inspeção era viável. Assim sendo, a pesquisa procedeu-se para todos os pares $(m ; a)$ de tal modo que $0 \leq m \leq 6, a=\lfloor b m\rfloor \mathrm{e}$ $0 \leq b<1$ como requerido pela proposição 3. A metodologia proposta por Quinino et al. (1999) define a pesquisa para os valores de $m$ tal que $0 \leq m \leq 83$, muito maior do que o da pesquisa realizada neste trabalho. Para o cálculo de $\left(\mathrm{m}^{\circ} ; a^{\circ}\right)$ realizamos implementação computacional no software estatístico MINITAB. O programa pode ser obtido através de solicitação direta com os autores.

A utilização do programa implementado em MINITAB forneceu o $m$ ótimo $\left(m^{\circ}\right)$ igual a 2 e o $a$ ótimo $\left(a^{\circ}\right)$ igual a 0 . Esta política de decisão indica que o produto será considerado conforme se pelo menos uma das duas classificações repetidas independentes julgar o produto conforme. O custo médio total desta política de decisão foi de $\$ 3.900,00$. Observe que o procedimento tradicional de classificar somente uma única vez produz um custo médio total de $\$ 9.200,00$, maior inclusive que o valor $\$ 6.000,00$ referente a não realizar qualquer plano de inspeção. Quinino et al. (1999) utilizando a política de inspeção com $m$ igual a 3 e o $a$ igual a 1 obtiveram o custo médio total de $\$ 5.296,00$. A Figura 1 mostra o comportamento de $E_{m, a}\left(p, e_{1}, e_{2}\right), \mathrm{m} \geq 0, \mathrm{a}=\mathrm{bm}$. A Tabela 1 explicita os valores pesquisados.

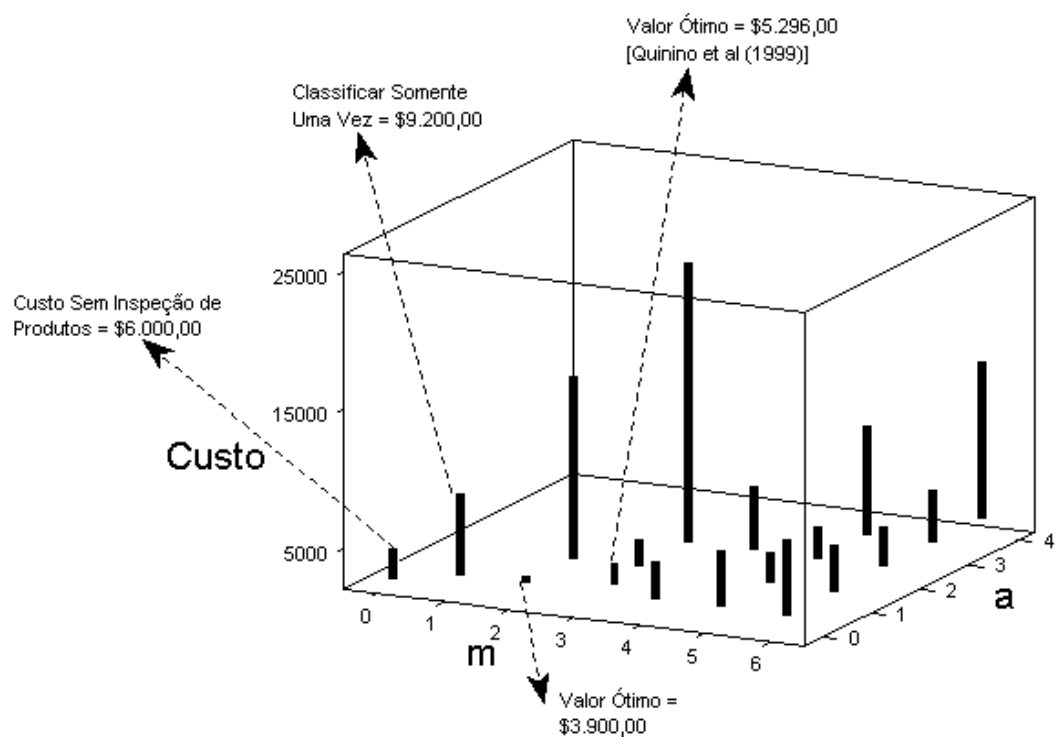

Figura 1 - Custo Médio Total versus "m" e "a" 
Tabela 1 - Valores pesquisados " $m$ " e " $a$ " versus custos

\begin{tabular}{|c|c|c|}
\hline Custo & \multicolumn{2}{|c|}{ Valores Pesquisados } \\
\hline$\$$ & $\mathrm{~m}$ & $\mathrm{a}$ \\
\hline $6.000,00$ & 0 & ${ }^{\star}$ \\
\hline $9.200,00$ & 1 & 0 \\
\hline $3.900,00$ & 2 & 0 \\
\hline $16.500,00$ & 2 & 1 \\
\hline $4.702,00$ & 3 & 0 \\
\hline $5.296,00$ & 3 & 1 \\
\hline $23.602,00$ & 3 & 2 \\
\hline $6.071,00$ & 4 & 0 \\
\hline $4.595,00$ & 4 & 1 \\
\hline $7.997,00$ & 4 & 2 \\
\hline $30.137,00$ & 4 & 3 \\
\hline $7.457,80$ & 5 & 0 \\
\hline $5.523,70$ & 5 & 1 \\
\hline $5.701,90$ & 5 & 2 \\
\hline $11.193,70$ & 5 & 3 \\
\hline $36.122,80$ & 5 & 4 \\
\hline $8.811,40$ & 6 & 0 \\
\hline $6.689,80$ & 6 & 1 \\
\hline $6.191,60$ & 6 & 2 \\
\hline $7.212,20$ & 6 & 3 \\
\hline $14.684,50$ & 6 & 4 \\
\hline $41.610,50$ & 6 & 5 \\
\hline & & \\
\hline
\end{tabular}

\section{Conclusão e Considerações Finais}

Erros de classificação podem causar um significativo impacto nas conclusões sobre a qualidade do processo de produção e conseqüentemente aumentar o custo médio total de todo o processo. Conforme este trabalho, realizar independentemente mais de uma classificação por produto fabricado pode minimizar o custo médio total de todo o processo. $\mathrm{O}$ modelo sugerido fornece o número ótimo de classificações repetidas por produto conjuntamente com o número ótimo de conformidades, a serem verificadas nas classificações repetidas, para julgar o produto conforme. As variáveis necessárias para implementação do modelo são de fácil obtenção nos processos de fabricação.

Sugestão de extensão deste trabalho pode ser realizada através da elaboração de uma nova estratégia de decisão. Esta consiste de realizar repetitivamente classificações independentes por produto até a verificação de $c$ (inteiro e $c \geq 1$ ) classificações conformes ou $d$ (inteiro e $d \geq 1$ ) classificações não-conformes. O produto será julgado conforme se for verificado $c$ classificações conformes ou será julgado não-conforme se for verificado $d$ classificações não-conformes. Tudo isto torna o modelo complicado. O processo de classificação e decisão pode ser modelado como uma Cadeia de Markov redutível com espaço de estados $(s, j)$, $s=0, \ldots, \mathrm{c} ; j=0, \ldots, \mathrm{d}$, absorventes ou transitórios. 


\section{Referências Bibliográficas}

(1) Burke, J.R.; Davis, R.D.; Kaminsky, F.C. \& Roberts, A.E.P. (1995). The effect of inspector errors on the true fraction non-conforming: an industrial experiment. Quality Engineering, 7, 543-550.

(2) Gramopadhye, A.K.; Pajjuri, V.G.; Kimbler, D. \& Ferrell Jr., W.G. (1995-96). Compensating for inspection errors in attribute inspection. Quality Engineering, 8(2), 311-22.

(3) Greenberg, B.S. \& Stokes, S.L. (1995). Repetitive testing in the presence of inspection errors. Technometrics, 37(1), 102-111.

(4) Johnson, N.L.; Kotz, S. \& Wu, X. (1991). Inspection errors for attributes in quality control. Chapman \& Hall, London.

(5) Kemp, A.W. \& Kemp, C.D. (1988). A simple inspection scheme for two types of defect. Journal of the Operational Research Society, 39(3), 311-15, 1988.

(6) Quinino, R.C.; Colin, E.C. \& Bueno Neto, P.R. (1999). Determinação do número ótimo de classificações imperfeitas na avaliação da conformidade de produtos. Gestão \& Produção, 6(2), 76-85. 